\title{
Clozapine's functional mesolimbic selectivity is not duplicated by the addition of anticholinergic action to haloperidol: a brain stimulation study in the rat
}

\author{
Eliot L. Gardner ${ }^{1,2}$, Leslie Stern Walker ${ }^{1, *}$, and William Paredes ${ }^{1}$ \\ Program in Brain and Behavior, ${ }^{1}$ Department of Psychiatry and ${ }^{2}$ Department of Neuroscience, \\ Albert Einstein College of Medicine, 1300 Morris Park Avenue, Bronx, NY 10461, USA
}

Received April 29, 1992 / Final version July 31, 1992

\begin{abstract}
This study examined whether the anticholinergic potency of the clinically superior antipsychotic drug clozapine contributes to clozapine's anatomically-selective functional inhibition of the mesolimbic dopamine (DA) system, using an electrical brain-stimulation reward (BSR) paradigm in rats that has been previously shown to be highly sensitive to clozapine's mesolimbic functional selectivity. Rats were chronically administered saline, clozapine, haloperidol, or haloperidol plus the anticholinergic compound trihexyphenidyl, and threshold sensitivity of the mesolimbic and nigrostriatal DA systems was assessed using the BSR paradigm, to infer degree of functional DA blockade produced by the chronic drug regimens. Chronic saline produced no change in either DA system. Congruent with previous findings, chronic clozapine powerfully inhibited the mesolimbic DA system but spared the nigrostriatal DA system. Also congruent with previous findings, chronic haloperidol powerfully inhibited both DA systems. Compared to chronic haloperidol alone, chronic haloperidol plus chronic trihexyphenidyl exerted diminished anti-DA action in both the mesolimbic and nigrostriatal DA systems. These results suggest that clozapine's anticholinergic potency is not an adequate explanation for its functional mesolimbic selectivity.
\end{abstract}

Key words: Clozapine - Haloperidol - Trihexyphenidyl - Dopamine - Acetylcholine - Mesolimbic - Nigrostriatal - Antipsychotic - Neuroleptic - Atypical Schizophrenia - Psychosis - Substantia nigra - Ventral tegmental area - Brain stimulation reward-Self-stimulation - ICSS

\footnotetext{
* Present address: Medical Scientist Training Program and Department of Neuroscience, University of Michigan, Ann Arbor, MI 48104, USA

Correspondence to: E.L. Gardner
}

The antipsychotic drug clozapine is remarkable. It is a superior antipsychotic agent, with proven therapeutic efficacy for even neuroleptic-resistant schizophrenia (Kane et al. 1988; Meltzer et al. 1989a; Gerlach 1991; Kane 1992). It is also virtually devoid of propensity to induce extrapyramidal side effects and tardive dyskinesia (Casey 1989; Meltzer et al. 1989a; Gerlach 1991; Jann 1991; Kane 1992). The neuropharmacological mechanisms underlying this unusual clinical profile are unclear (Coward et al. 1989; Meltzer et al. 1989a; Gerlach 1991; Jann 1991). While clozapine's interaction with classically defined brain dopamine (DA) receptors (i.e., $\mathrm{D}_{1}$ and $\mathrm{D}_{2}$ ) is weak, studies of presynaptic DA neural function rather than postsynaptic DA receptor occupancy (e.g., behavioral, electrophysiological recording, electrical brain stimulation, in vivo brain microdialysis, and in vivo brain electrochemistry studies) show that clozapine powerfully inhibits mesolimbic DA function while sparing nigrostriatal DA function (Huff and Adams 1980; Chiodo and Bunney 1983; White and Wang 1983; Hand et al. 1987; Gardner and Seeger 1988; Chen et al. 1991; Gardner 1992a). This selective action on the mesolimbic system may partly account for clozapine's atypical clinical profile, since the antipsychotic efficacy of neuroleptics is widely presumed to derive from suppressive action on the mesolimbic DA system (Stevens 1979; Creese 1983) while their extrapyramidal side effects are presumed to derive from suppressive action on the nigrostriatal DA system (Creese 1983; Baldessarini 1985).

Clozapine affects many brain transmitter systems (Meltzer et al. 1989a; Gerlach 1991; Jann 1991), but which of clozapine's neuropharmacological actions imparts its anatomically selective functional antagonism of the mesolimbic DA system is obscure. Suggestions have included clozapine's serotonergic blockade (Meltzer et al. 1989a; Chen et al. 1992b), noradrenergic blockade (Chiodo and Bunney 1985), preferential blockade of $D_{1}$ versus $D_{2}$ receptors (Coward et al. 1989), combined blockade of $D_{1}$ and $D_{2}$ receptors (Farde et al. 1989), interaction with a new subtype of DA receptor (van Tol et al. 1991), and production of specific ratios of blockade 
between multiple neurotransmitter systems (Meltzer et al. 1989b). Recently, two groups (Chiodo and Bunney 1985; Lane and Blaha 1986) have reported that, in rats, the addition of an anticholinergic to the typical neuroleptic haloperidol changes haloperidol's nonselective functional inhibition of both the mesolimbic and nigrostriatal DA systems into an anatomically selective inhibition of the mesolimbic DA system resembling that of clozapine. Since clozapine is potently anticholinergic (Bolden et al. 1992), such findings have initial appeal. However, they appear at variance with the observations that: 1) addition of anticholinergics to neuroleptic medication $d e-$ tracts from antipsychotic efficacy rather than producing the superior antipsychotic effects of clozapine (Singh and Kay 1979; Johnstone et al. 1983), and 2) anticholinergic medications do not reduce the risk of tardive dyskinesia after long-term neuroleptic exposure (Klawans 1973) and can even exacerbate existing tardive dyskinesia (Baldessarini and Tarsy 1979) rather than producing the suppression of tardive dyskinesia seen with clozapine (Casey 1989; Kane 1992).

We have previously adapted the paradigm of electrical brain-stimulation reward (BSR) as an anatomically specific probe of functional DA activation or inhibition in the mesolimbic and nigrostriatal DA systems. This approach rests upon the coincidence that portions of the mesolimbic and nigrostriatal DA systems of the mammalian brain are involved in mediating direct brain reward (Wise and Rompre 1989; Gardner 1992b). Using the BSR paradigm, we and others have found that chronic DA antagonist administration produces robust and prolonged functional DA upregulation following antagonist cessation, reflecting a rebound functional DA supersensitivity (Ettenberg and Milner 1977; Seeger and Gardner 1979; Seeger et al. 1981). This paradigm is highly sensitive to the functional DA blockade produced by antipsychotic drugs, and to antipsychotic drug dose (Seeger and Gardner 1979; Seeger et al. 1981; Gardner and Seeger 1988; Gardner 1992a). Using this paradigm in animals with electrodes implanted into mesolimbic and nigrostriatal DA loci, we have previously reported that BSR is highly sensitive to the mesolimbically-selective functional DA antagonism produced by clozapine (Gardner and Seeger 1988; Gardner 1992a). Specifically, in animals with electrodes implanted in the mesolimbic system, both haloperidol and clozapine (in chronic doses equated for antischizophrenic efficacy) produced highly significant (and equivalent) BSR enhancement following chronic treatment. In animals implanted with electrodes in the nigrostriatal system, only haloperidol produced BSR enhancement following chronic treatment, while clozapine's effects were indistinguishable from those of chronic saline (Gardner and Seeger 1988; Gardner 1992a).

Given this utility of the BSR paradigm to detect clozapine's mesolimbically-selective action, the present study was undertaken to determine whether the addition of the potent anticholinergic compound trihexyphenidyl to a regimen of chronic haloperidol would shift haloperidol's non-selective functional blockade of both the mesolimbic and nigrostriatal DA systems to a meso- limbically selective functional DA blockade resembling that of clozapine.

\section{Materials and methods}

Forty male Sprague-Dawley rats (Taconic) weighing between 200 and $225 \mathrm{~g}$ at the start of experimentation were used. They were housed individually on ad libitum food and water in an animal colony room kept at $22^{\circ} \mathrm{C}$ with a $12 \mathrm{~h}$ light-dark cycle. Rats were randomly assigned to the following four chronic drug groups: saline, clozapine, haloperidol, and haloperidol plus trihexyphenidyl. The rats were then anesthetized with sodium pentobarbital $(50 \mathrm{mg} / \mathrm{kg})$ and surgically implanted, using standard stereotaxic procedures, with chronic bipolar intracranial BSR electrodes (Plastic Products type MS303/1). Half of the animals in each drug group were implanted with electrodes in the ventral tegmental area (DA nucleus A10; mesolimbic DA cell bodies of origin) at stereotaxic coordinates AP $+2.8, \mathrm{~L} 1.2, \mathrm{H}-3.5$, according to the rat brain stereotaxic atlas of Pellegrino et al. (1979). The other half of each drug group were implanted with electrodes in the substantia nigra (DA nucleus A9; nigrostriatal DA cell bodies of origin) at stereotaxic coordinates AP $+2.8, \mathrm{~L} 2.3, \mathrm{H}-2.8$ (Pellegrino et al. 1979). The animals were allowed 10 days recovery from surgery and were then trained to self-administer BSR electrical stimulation through the implanted electrodes using methods we have previously described (Gardner et al. 1988). Training and testing was carried out in standard operant chambers equipped with two response levers. Microprocessor/brain-stimulator units were programmed to deliver titrating threshold decremental brain stimulation. Each response by the animal on the primary lever delivered a $250 \mathrm{~ms}$ stimulus train ( $60 \mathrm{~Hz}$ bipolar rectangular pulse-pairs of $0.3 \mathrm{~ms}$ duration, with a $0.15 \mathrm{~ms}$ delay between the pulses of each balanced pair of opposite polarity) through the brain electrode. Initial current intensity was assigned to each animal at the lowest intensity supporting reliable responding at a rate of 650 lever presses per 20-min test session. Training was initiated using continuous (fixed ratio 1:1) BSR reinforcement on the primary lever. This was followed by training sessions in which first one lever and then the other was active, to induce the rats to alternate between levers to seek the stimulusactive lever. After proficiency and high stable rates of responding were achieved at this task, the animals were switched to the final BSR threshold titration paradigm. For this, the primary lever was fixed as the only lever delivering BSR, and the previously assigned stimulation current level for each individual rat was set as the maximum level of a descending series of current intensity steps for that animal. The BSR current decremented by $1 / 16$ of this maximum intensity at every third press of the primary lever. At any time during the ensuing sequence of BSR stimuli of decreasing magnitude, the animal could reset the current to its individually-assigned maximum by pressing the second or "reset" lever, which reset the brain stimulation back to maximum for that animal and also activated a cue-signal tone but did not itself deliver brain stimulation. The current steps at which each animal reset were automatically recorded throughout each 20 -min test session, and the mean of the resulting frequency distribution of reset levels was operationally defined as the BSR threshold. The standard deviation of this frequency distribution was taken as a measure of performance stability, with a standard deviation of \pm 1.5 current steps used as the criterion for stable performance and inclusion in the drug study. Brain stimulation threshold data were collected and analyzed by on-line microprocessor units. After training to stable performance, each animal was tested daily for a minimum of 6 weeks to ensure an absolutely stable baseline of BSR thresholds before drug trials began.

The drugs and amounts used were: haloperidol, $1.0 \mathrm{mg} / \mathrm{kg} / \mathrm{day}$; clozapine, $20.0 \mathrm{mg} / \mathrm{kg} / \mathrm{day}$; trihexyphenidyl hydrochloride, $1.0 \mathrm{mg} /$ $\mathrm{kg} /$ day; saline, $0.4 \mathrm{ml} /$ day. The clozapine was dissolved in dilute hydrochloric acid and adjusted to $\mathrm{pH} 3.5$ with sodium bicarbonate. The clozapine dose was chosen by a comprehensive survey of 
clozapine use, both clinically and in electrophysiological, biochemi$\mathrm{cal}$, and behavioral laboratory paradigms. The haloperidol dose was similarly chosen. These are the same doses used in our previous studies of clozapine's functional mesolimbic DA selectivity (Seeger and Gardner 1979; Seeger et al. 1981; Gardner and Seeger 1988; Chen et al. 1991; Chen et al. 1992a,b; Gardner 1992a). The 20:1 dose ratio between clozapine and haloperidol also reflects the approximate clinical ratio needed for equivalent control of schizophrenia (Seeman 1987); it is also the same dose ratio used by other groups studying clozapine's mesolimbic selectivity (Chiodo and Bunney 1985). Trihexyphenidyl's anticholinergic profile resembles that of clozapine (Bolden et al. 1992). The trihexyphenidyl dose was chosen as representing a clinically meaningful ratio to the haloperidol dose, and is the same dose used by Chiodo and Bunney (1985) and Lane and Blaha (1986) in their studies of anticholinergic involvement in clozapine's mesolimbic selectivity.

After the 6-week BSR stabilization period, pre-drug baseline BSR threshold level was determined for each rat on three consecutive days, after which each rat was given the drug treatment (by intraperitoneal injection, once daily at $4: 00 \mathrm{pm}$ for 3 weeks) corresponding to its assigned group (clozapine, haloperidol, haloperidol plus trihexyphenidyl, saline). We have previously shown that 3 weeks of daily antipsychotic treatment yields maximal functional DA upregulation in the BSR paradigm (Seeger and Gardner 1979; Seeger et al. 1981; Gardner and Seeger 1988; Gardner 1992a). After the chronic drug treatment, rats were given 3 days of drug washout, and then retested in the BSR paradigm for drug-induced functional hypersensitivity in the mesolimbic or nigrostriatal DA system at 3 , $5,7,10,13,16,19,22,25,28$, and 32 days following termination of drug treatment. Significance of shifts in BSR threshold between predrug and postdrug test sessions, and between drug groups, was analyzed by analysis of variance (ANOVA) for repeated measures (Kirk 1982), with individual comparisons within each data set tested for significance by the Tukey-Kramer a posteriori statistical procedure (Kirk 1982).

At the end of the study, all animals were killed by anesthetic overdose, the brains removed, and standard histology carried out to confirm the brain locations of the BSR electrodes.

\section{Results}

Three weeks of saline had no effect on BSR thresholds from electrodes in either the mesolimbic or nigrostriatal DA systems. In animals with mesolimbic electrodes, both chronic haloperidol and chronic clozapine produced significantly lower BSR thresholds following cessation of antipsychotic drug treatment (ANOVA for repeated measures: main drugs effect $-F_{2,11}=8.2, P<0.01$; main test sessions effect $-F_{10,110}=88.2, P<0.0001$; drugs $\times$ test sessions interaction $\left.-F_{20,110}=12.5, P<0.0001\right)$. In these mesolimbically implanted animals, individual comparisons showed that BSR thresholds following both chronic haloperidol and chronic clozapine were significantly enhanced as compared to BSR thresholds following chronic saline for days $3,5,7,10,13$, and 16 after cessation of drug treatment.

In animals with nigrostriatal electrodes, only chronic haloperidol produced significantly lowered BSR thresholds following cessation of antipsychotic drug treatment (ANOVA for repeated measures: main drugs effect $F_{2,10}=7.8, \quad P<0.01$; main test sessions effect $F_{10,100}=17.2, P<0.0001$; drugs $\times$ test sessions interaction $\left.-F_{20,100}=11.4, P<0.0001\right)$. In the nigrostriatal system, the effect of chronic clozapine was no different from that of saline. Individual comparisons of the data
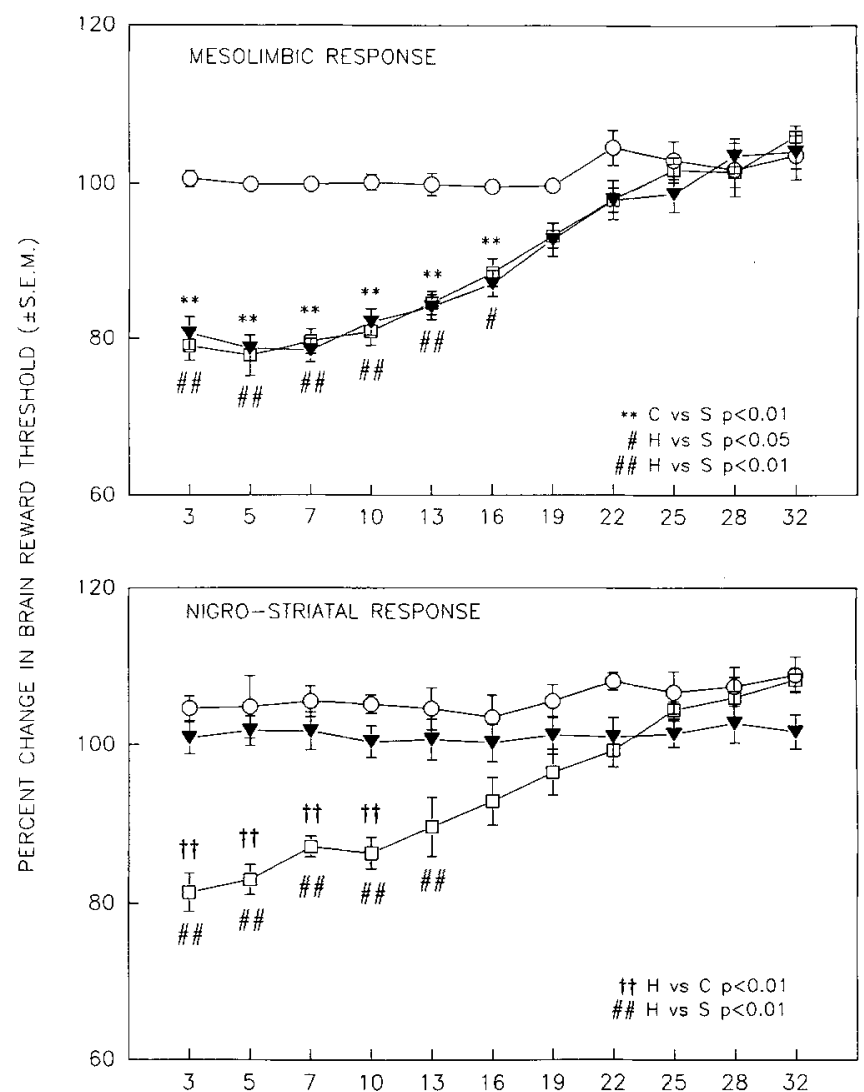

DAYS AFTER LAST DRUG TREATMENT

Fig. 1. Effects of 3 weeks of chronic daily treatment with saline $(O)$,

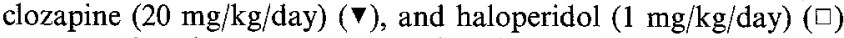
on post-chronic-treatment DA functional upregulation in the mesolimbic and nigrostriatal DA systems, as assessed by electrical brain-stimulation reward thresholds in laboratory rats. Decreases in brain reward threshold (i.e., data points significantly below the $100 \%$ baseline) represent enhanced DA functional upregulation following cessation of chronic drug treatment, implying functional DA antagonism during chronic drug treatment (see text). The statistically significant individual comparisons noted on the figure by $\dagger \uparrow$, **, \#, and \#\# were calculated using the Tukey-Kramer a posteriori statistic, following overall statistically significant ANOVAs for repeated measures (see text)

from nigrostriatally-implanted animals showed that BSR thresholds following chronic haloperidol were significantly enhanced as compared to BSR thresholds following both chronic saline and chronic clozapine for days 3, 5, 7, 10,13 and for days $3,5,7,10$, respectively, after cessation of drug treatment. BSR thresholds following chronic clozapine were not significantly different from BSR thresholds following chronic saline at any point. These findings are illustrated in Fig. 1.

The addition of trihexyphenidyl to haloperidol detracted from haloperidol's potency in both the mesolimbic and nigrostriatal systems. In aminals with mesolimbic electrodes, chronic haloperidol produced significantly lower BSR thresholds following cessation of antipsychotic drug treatment than did the chronic haloperidol plus trihexyphenidyl regimen, but the haloperidol plus trihexyphenidyl did not significantly lower BSR thresholds following cessation of antipsychotic drug 

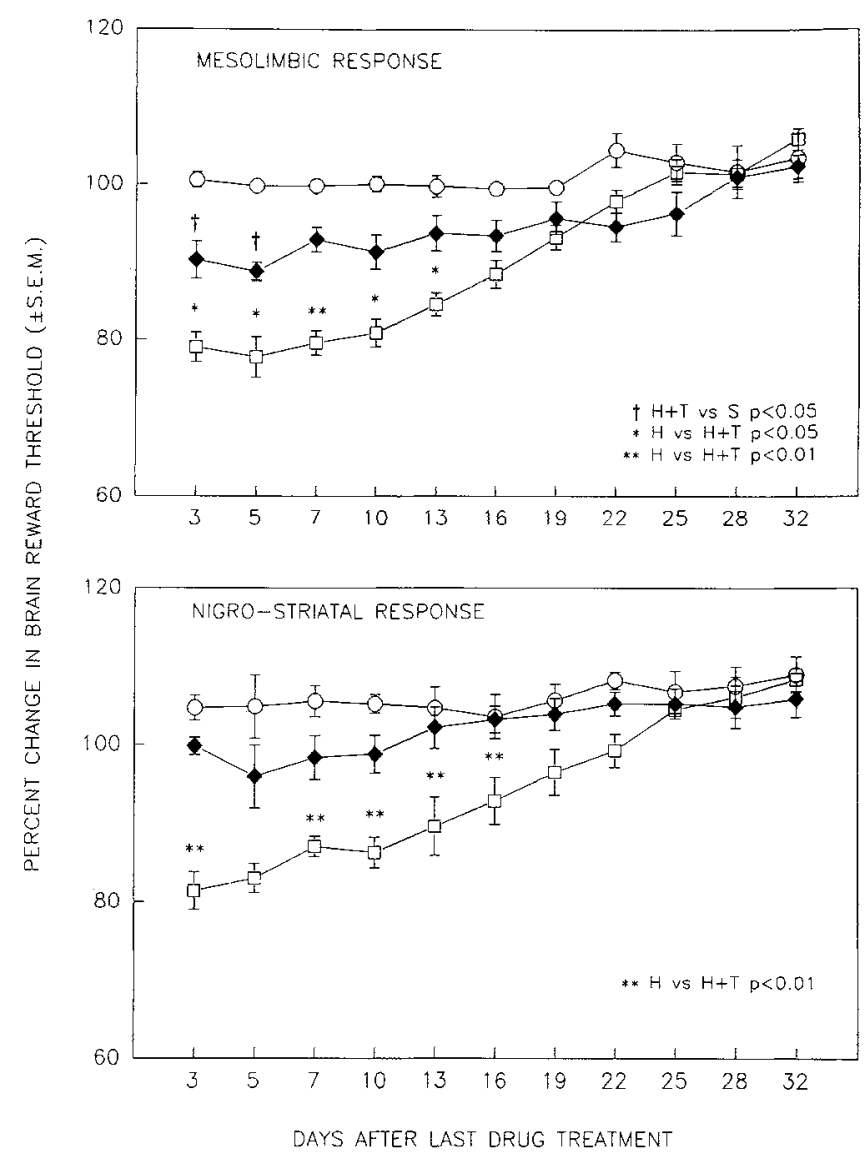

Fig. 2. Effects of 3 weeks of chronic daily treatment with saline $(O)$, haloperidol ( $1 \mathrm{mg} / \mathrm{kg} /$ day $)(\square)$, and haloperidol-plus-trihexyphenidyl $(1 \mathrm{mg} / \mathrm{kg} /$ day plus $1 \mathrm{mg} / \mathrm{kg} /$ day) (*) on post-chronic-treatment DA functional upregulation in the mesolimbic and nigrostriatal DA systems, as assessed by electrical brain-stimulation reward thresholds in laboratory rats. Decreases in brain reward threshold (i.e., data points significantly below the $100 \%$ baseline) represent enhanced DA functional upregulation following cessation of chronic drug treatment, implying functional DA antagonism during chronic drug treatment (see text). The statistically significant individual comparisons noted on the figure by,$+ *$, and ** were calculated using the Tukey-Kramer a posteriori statistic, following overall statistically significant ANOVAs for repeated measures (see text). The saline and haloperidol groups shown here are the same animals as shown for those groups in Fig. 1

treatment as compared to saline (overall ANOVA for repeated measures: main drugs effect $-F_{2,12}=9.1$, $P<0.005$; main test sessions effect $-F_{10,120}=31.7$, $P<0.0001$; drugs $\times$ test sessions interaction $\left.F_{20,120}=10.3, P<0.0001\right)$. In these mesolimbically implanted animals, individual comparisons showed that BSR thresholds following chronic haloperidol were significantly enhanced as compared to BSR thresholds following chronic haloperidol plus trihexyphenidyl for days $3,5,7,10$, and 13 after cessation of drug treatment. BSR thresholds following chronic haloperidol plus trihexyphenidyl were not significantly different from BSR thresholds following chronic saline at any point, except for the first 2 days after cessation of antipsychotic drug treatment. Similarly, in animals with nigrostriatal electrodes, chronic haloperidol produced significantly lower BSR thresholds following cessation of antipsychotic drug treatment than did the chronic haloperidol plus trihexyphenidyl regimen, but the chronic haloperidol plus trihexyphenidyl combination did not significantly lower BSR thresholds following cessation of antipsychotic drug treatment as compared to saline (overall ANOVA for repeated measures: main drugs effect - $F_{2,10}=11.9, P<0.005$; main test sessions effect $F_{10,100}=14.6, P<0.0001$; drugs $\times$ test sessions interaction $-F_{20,100}=4.4, P<0.0001$ ). In these nigrostriatally implanted animals, individual comparisons showed that BSR thresholds following chronic haloperidol were significantly enhanced as compared to BSR thresholds following chronic haloperidol plus trihexyphenidyl for days $3,7,10,13$, and 16 after cessation of drug treatment. BSR thresholds following chronic haloperidol plus trihexyphenidyl were not significantly different from BSR thresholds following chronic saline at any point. These findings are illustrated in Fig. 2.

\section{Discussion}

The present findings suggest several things. First, they confirm the notable mesolimbic anatomic selectivity of clozapine's functional DA blockade. Chronic neuroleptic treatment is well known to produce DA functional upregulation following cessation of neuroleptic treatment, similar to the DA denervation supersensitivity seen after lesions of central DA pathways (Ungerstedt 1971; Heal et al. 1976). The BSR paradigm is a sensitive assay of this DA functional upregulation following chronic neuroleptic treatment (Ertenberg and Milner 1977; Seeger and Gardner 1979; Seeger et al. 1981), from which it is inferred that functional DA blockade occurred during the neuroleptic treatment. Also, the BSR paradigm is sensitive to clozapine's anatomically selective functional blockade of the mesolimbic DA system (Gardner and Seeger 1988; Gardner 1992a). The present data thus agree with such previous BSR studies, and also agree with studies of clozapine's mesolimbically selective DA blockade using other paradigms (e.g., Huff and Adams 1980; Chiodo and Bunney 1983; White and Wang 1983; Hand et al. 1987; Chen et al. 1991). Interestingly, clozapine's mesolimbic selectivity is far more pronounced with chronic treatment than with acute (Gardner 1992a). Also of interest is the fact that only a subset of the mesolimbic DA system is affected by clozapine - the mesolimbic DA fibers terminating subcortically are blocked by clozapine while those terminating in the medial prefrontal cortex are not (Chen et al. 1991, 1992a). This mesolimbically selective DA blockade induced by chronic clozapine appears to be depolarization blockade (Chiodo and Bunney 1985; Blaha and Lane 1987; Chen et al. 1991).

The extent to which clozapine's mesolimbic selectivity is duplicated by other clinically atypical antipsychotic drugs is not clear. The antipsychotics commonly referred to as "atypical" have widely differing preclinical and clinical profiles. Included are such "partially atypical" phenothiazines as thioridazine and mesoridazine, such "partially atypical" benzamides as sulpiride and remoxipride, and a number of other "partially atypical" compounds of widely varying chemical structures and receptor specificities. They do not constitute a homogeneous pharmacological class by any clinical or preclinical 
criteria, and have each acquired the "atypical" label by exclusion rather than inclusion, i.e., by producing clinical effects or side effects different from those produced by classical neuroleptics (yet also often significantly different from each other). None of these "partially atypical" antipsychotics has been shown to fully duplicate clozapine's clinical profile. Yet, in spite of their exceedingly heterogenous nature, a number of them partially share clozapine's mesolimbic selectivity (Gardner 1992a). Unfortunately, the newest and most clozapinelike atypical drugs such as risperidone have not been well characterized in terms of mesolimbic/nigrostriatal selectivity.

Second, the present data call into question the adequacy of invoking clozapine's anticholinergic potency to explain its mesolimbic selectivity. In the present study, the addition of the anticholinergic drug trihexyphenidyl to chronic haloperidol detracted from haloperidol's functional DA blockade in both the mesolimbic and nigrostriatal DA systems. These findings are at variance with those of Chiodo and Bunney (1985) and Lane and Blaha (1986), who found, using single-neuron electrophysiological recording and in vivo voltammetry techniques, respectively, that the addition of trihexyphenidyl to chronic haloperidol detracted from haloperidol's functional DA blockade in only the nigrostriatal DA system, thus mimicking clozapine's profile. It is not clear why the present data are at variance with those of Chiodo and Bunney (1985) and Lane and Blaha (1986), beyond the obvious and perhaps trivial explanation that we used a different laboratory approach to inferring anatomically selective functional DA blockade produced by antipsychotic drug treatment. On the other hand, the present data are clearly more congruent with the clinically observed phenomena that the addition of anticholinergic medication to neuroleptic therapy not only detracts from nigrostriatal functional DA blockade (i.e., ameliorates neurolepticinduced extrapyramidal side effects) but also detracts from mesolimbic functional DA blockade (i.e., decreases antipsychotic efficacy) (Singh and Kay 1979; Johnstone et al. 1983).

In considering the present findings, the comparative anticholinergic profiles of clozapine and trihexyphenidyl should be noted. Using pharmacological approaches with selective antagonists, three muscarinic receptor subtypes, respectively designated $\mathrm{M}_{1}, \mathrm{M}_{2}$, and $\mathrm{M}_{3}$, have been identified (Mitchelson 1988). By such pharmacological differentiation, both clozapine and trihexyphenidyl are relatively selective $\mathrm{M}_{1}$ antagonists. Using molecular biological approaches, five human muscarinic receptor subtypes - designated $\mathrm{m} 1, \mathrm{~m} 2, \mathrm{~m} 3, \mathrm{~m} 4$, and $\mathrm{m} 5$ - have been cloned and shown to be expressed in brain and periphery (Bolden et al. 1992). It is believed that the first three of these gene products - the $\mathrm{m} 1, \mathrm{~m} 2$, and $\mathrm{m} 3$ entities - correspond, respectively, to the pharmacologically identified $\mathrm{M}_{1}, \mathrm{M}_{2}$, and $\mathrm{M}_{3}$ receptor subtypes (Bolden et al. 1992). Both clozapine and trihexyphenidyl interact with all five cloned muscarinic receptor subtypes, but show high selectivity for the m1 subtype (Bolden et al. 1992). They also show secondary selectivity for the $\mathrm{m} 4$ subtype. While not precisely duplicating clozapine's anticholinergic profile, trihexyphenidyl resembles clozapine reasonably well. In any event, slight differences in the antimuscarinic profiles of clozapine and trihexyphenidyl would appear irrelevant to any hypothesis for the differences between the present findings and those of Chiodo and Bunney (1985) and Lane and Blaha (1986), since the present study utilized the same reference anticholinergic (trihexyphenidyl), and precisely the same dose of trihexyphenidyl, as those workers.

The present conclusion that clozapine's anticholinergic effects are not an adequate explanation for its mesolimbic selectivity and atypical clinical profile is supported by previous work (Coward et al. 1989), including animal studies showing that combining haloperidol with atropine only partly mimics clozapine's pharmacological profile (Sayers et al. 1976). If clozapine's anticholinergic action does not explain its mesolimbic selectivity, what does? The possibilities are legion, since clozapine interacts with most if not all conventional neurotransmitters (Coward et al. 1989). Among the possibilities, however, current evidence suggests an important serotonin (5HT) component (Meltzer et al. 1989b; Chen et al. 1992b). Clozapine's antagonist potency is far more pronounced at $5 \mathrm{HT}$ receptors than at classically defined DA receptors. In addition, 5HT fibers innervate and powerfully modulate both the mesolimbic and nigrostriatal DA systems (Hetey and Drescher 1986; De Simoni et al. 1987). However, the mesolimbic DA system receives a denser $5 \mathrm{HT}$ innervation than does the nigrostriatal DA system, and $5 \mathrm{HT}_{1 \mathrm{~B}}, 5 \mathrm{HT}_{2}$, and $5 \mathrm{HT}_{3}$ receptors are all more densely distributed in the mesolimbic DA system than the nigrostriatal DA system (Pazos and Palacios 1985; Fischette et al. 1987; Waeber et al. 1988). Thus, the preferential mesolimbic distribution of 5HT afferents and receptors, coupled with 5 HT's powerful modulatory regulation of forebrain DA function and clozapine's potent 5HT antagonism, may partly explain clozapine's preferential inhibition of the mesolimbic DA system (Chen et al. 1992b).

Finally, the present data suggest that, in the absence of counteracting properties, the anticholinergic actions of antipsychotic drugs or drug combinations can be expected to detract from antipsychotic efficacy.

Acknowledgements. This work was supported by National Institute of Mental Health research grant MH 43619 and by Biomedical Research Support grant RR 05397 from the National Institutes of Health, US Public Health Service. We thank the Sandoz Research Institute of Sandoz Pharmaceuticals Corporation for donating the clozapine. We also thank Dr. Herman M. van Praag, Chairman, Department of Psychiatry, and Dr. Dominick P. Purpura, Dean, Albert Einstein College of Medicine, for their support. L.S.W. was an undergraduate research scholar of the Sue Golding Graduate Division of Medical Sciences, Albert Einstein College of Medicine.

\section{References}

Baldessarini RJ (1985) Chemotherapy in psychiatry : principles and practice, 2nd edn. Harvard University Press, Cambridge

Baldessarini RJ, Tarsy D (1979) Relationship of the actions of neuroleptic drugs to the pathophysiology of tardive dyskinesia. Int Rev Neurobiol 21:1-45

Blaha CD, Lane RF (1987) Chronic treatment with classical and atypical antipsychotic drugs differentially decreases dopamine release in striatum and nucleus accumbens in vivo. Neurosci Lett 78:199-204

Bolden C, Cusack B, Richelson E (1992) Antagonism by antimuscarinic and neuroleptic compounds at the five cloned human 
muscarinic cholinergic receptors expressed in Chinese hamster ovary cells. J Pharmacol Exp Ther 260:576-580

Casey DE (1989) Clozapine: neuroleptic-induced EPS and tardive dyskinesia. Psychopharmacology 99[suppl]:S47-S53

Chen J, Paredes W, Gardner EL (1991) Chronic treatment with clozapine selectively decreases basal dopamine release in nucleus accumbens but not in caudate-putamen as measured by in vivo brain microdialysis: further evidence for depolarization block. Neurosci Lett 122:127-131

Chen J, Ruan D, Paredes W, Gardner EL (1992a) Effects of acute and chronic clozapine on dopaminergic function in medial prefrontal cortex of awake, freely moving rats. Brain Res $571: 235-241$

Chen J, Paredes W, van Praag HM, Gardner EL (1992b) Serotonin denervation enhances responsiveness of presynaptic dopamine efflux to acute clozapine in nucleus accumbens but not in caudate-putamen. Brain Res 582:173-179

Chiodo LA, Bunney SB (1983) Typical and atypical neuroleptics: differential effects of chronic administration on the activity of A9 and A10 midbrain dopaminergic neurons. J Neurosci 3:1607-1619

Chiodo LA, Bunney SB (1985) Possible mechanisms by which repeated clozapine administration differentially affects the activity of two subpopulations of midbrain dopamine neurons. J Neurosei 5:2539-2544

Coward DM, Imperato A, Urwyler S, White TG (1989) Biochemical and behavioural properties of clozapine. Psychopharmacology 99 [suppl]: S6-S12

Creese I (1983) Classical and atypical antipsychotic drugs: new insights. TINS 6:479-481

De Simoni MG, Dal Toso G, Fodritto F, Sokola A, Algeri S (1987) Modulation of striatal dopamine metabolism by the activity of dorsal raphe serotonergic afferences. Brain Res 411:81-88

Ettenberg A, Milner PM (1977) Effects of dopamine supersensitivity on lateral hypothalamic self-stimulation in rats. Pharmacol Biochem Behav 7:507-514

Farde L, Wiesel FA, Nordström A-L, Sedvall G (1989) $D_{1}$ - and $\mathrm{D}_{2}$-dopamine receptor occupancy during treatment with conventional and atypical neuroleptics. Psychopharmacology 99[suppl]: S28-S31

Fischette CT, Nock B, Renner K (1987) Effects of 5,7-dihydroxytryptamine on serotonin ${ }_{1}$ and serotonin $_{2}$ receptors throughout the rat central nervous system using quantitative autoradiography. Brain Res 421:263-279

Gardner EL (1992a) Neuroanatomical specificity of action of the atypical antipsychotics: implications for a new era in the pharmacotherapy of schizophrenia. In: Lindenmayer J-P, Kay SR (eds) New biological vistas on schizophrenia. Bruner/Mazel, New York

Gardner EL (1992b) Brain reward mechanisms. In: Lowinson JH, Ruiz P, Millman RB (eds) Substance abuse: a comprehensive textbook, 2nd edn. Williams \& Wilkins, Baltimore

Gardner EL, Seeger TF (1988) Anatomically selective action of atypical neuroleptics on the mesocorticolimbic dopamine system. Ann NY Acad Sci 537:502-504

Gardner EL, Paredes W, Smith D, Donner A, Milling C, Cohen D, Morrison D (1988) Facilitation of brain stimulation reward by $\Delta^{9}$-tetrahydrocannabinol. Psychopharmacology 96:142-144

Gerlach J (1991) New antipsychotics: classification, efficacy, and adverse effects. Schizophr Bul1 17:289-309

Hand TH, Hu X-T, Wang RY (1987) Differential effects of acute clozapine and haloperidol on the activity of ventral tegmental (A10) and nigrostriatal (A9) dopamine neurons. Brain Res 415:257-269

Heal DJ, Green AR, Bullin DJ, Grahame-Smith DG (1976) Single and repeated administration of neuroleptic drugs to rats: effects on striatal dopamine-sensitive adenylate cyclase and locomotor activity. Psychopharmacology 49:287-300
Hetey L, Drescher K (1986) Influence of antipsychotics on presynaptic receptors modulating the release of dopamine in synaptosomes of the nucleus accumbens of rats. Neuropharmacology 25:1103-1109

Huff RM, Adams RN (1980) Dopamine release in n. accumbens and striatum by clozapine: simultaneous monitoring by in vivo electrochemistry. Neuropharmacology 19:587-590

Jann MW (1991) Clozapine. Pharmacotherapy 11:179-195

Johnstone EC, Crow TJ, Ferrier IN, Firth CD, Owens DGC, Bourne RC, Gamble SJ (1983) Adverse effects of anticholinergic medication on positive schizophrenic symptoms. Psychol Med $13: 513-527$

Kane J (1992) Atypical neuroleptics in the treatment of schizophrenia. In: Lindenmayer J-P, Kay SR (eds) New biological vistas on schizophrenia. Bruner/Mazel, New York

Kane J, Honigfeld G, Singer J, Meltzer H, et al. (1988) Clozapine for the treatment-resistant schizophrenic: a double-blind comparison with chlorpromazine. Arch Gen Psychiatry 45:789-796

Kirk RE (1982) Experimental design, 2nd edn. Brooks/Cole, Monterey, CA

Klawans HL (1973) The pharmacology of tardive dyskinesias. Am J Psychiatry 130:82-86

Lane RF, Blaha CD (1986) Electrochemistry in vivo: application to CNS pharmacology. Ann NY Acad Sci 473:50-69

Meltzer HY, Bastani B, Ramirez L, Matsubara S (1989a) Clozapine: new research on efficacy and mechanism of action. Eur Arch Psychiatr Neurol Sci 238:332-339

Meltzer HY, Matsubara S, Lee JC (1989b) The ratios of serotonin and dopamine ${ }_{2}$ affinities differentiate atypical and typical antipsychotic drugs. Psychopharmacol Bull 25:390-392

Mitchelson F (1988) Muscarinic receptor differentiation. Pharmacol Ther 37:357-423

Pazos A, Palacios IM (1985) Quantitative autoradiographic mapping of serotonin receptors in the rat brain. I. Serotonin-1 receptors. Brain Res 346:205-230

Pellegrino LJ, Pellegrino AS, Cushman AJ (1979) A stereotaxic atlas of the rat brain. Plenum Press, New York

Sayers AC, Bürki HR, Ruch W, Asper H (1976) Anticholinergic properties of antipsychotic drugs and their relation to extrapyramidal side-effects. Psychopharmacology 51:15-22

Seeger TF, Gardner EL (1979) Enhancement of self-stimulation behavior in rats and monkeys after chronic neuroleptic treatment: evidence for mesolimbic supersensitivity. Brain Res $175: 49-57$

Seeger TF, Gardner EL, Bridger WF (1981) Increase in mesolimbic electrical self-stimulation after chronic haloperidol: reversal by L-dopa or lithium. Brain Res 21 5:404-409

Seeman P (1987) Dopamine receptors and the dopamine hypothesis of schizophrenia. Synapse 1:133-152

Singh MM, Kay SR (1979) Therapeutic antagonism between anticholinergic anti-Parkinsonian agents and neuroleptics in schizophrenia: implications for a neuropharmacological model. Neuropsychobiology 5:74-86

Stevens RC (1979) Schizophrenia and dopamine dysregulation in the mesolimbic system. TINS 2:102-105

Ungerstedt U (1971) Postsynaptic supersensitivity after 6-hydroxydopamine induced degeneration of the nigrostriatal dopamine system. Acta Physiol Scand 82[suppl 367]:69-93

van Tol HH, Bunzow JR, Guan HC, Sunahara RK, Seeman P, Niznik HB, Civelli O (1991) Cloning of the gene for a human dopamine D4 receptor with high affinity for the antipsychotic clozapine. Nature 350: 610-614

Waeber C, Dixon K, Hoyer D, Palacios J M (1988) Localisation by autoradiography of neuronal $5-\mathrm{HT}_{3}$ receptors in the mouse CNS. Eur J Pharmacol 151:351-352

White FJ, Wang RY (1983) Differential effects of classical and atypical antipsychotic drugs on $\mathrm{A} 9$ and $\mathrm{A} 10$ dopamine neurons. Science $221: 1054-1057$

Wise RA, Rompre P-P (1989) Brain dopamine and reward. Annu Rev Psychol 40:191-225 